\title{
Hollow Core Fiber with an Octave Spanning Bandgap
}

\author{
Francesco Poletti \\ *Corresponding author: frap@orc.soton.ac.uk
}

\begin{abstract}
We thoroughly compare the out-of-plane bandgaps generated by three realistic two-dimensional lattices: a triangular and a square arrangement of holes and a triangular arrangement of rods. We demonstrate that for any given hole diameter to pitch ratio $\mathrm{d} / \Lambda$, the triangular arrangement of interconnected resonators generates the widest possible bandgap along the air line and we propose a physical interpretation explaining these results. The design of a hollow core photonic bandgap fiber based on such a lattice and able to transmit light with $\mathrm{sub} \mathrm{dB} / \mathrm{m}$ losses over an octave of frequencies is presented for the first time.
\end{abstract}

OCIS codes: (060.2280) Fiber design and fabrication; (060.5295) Photonic crystal fibers;

(160.5293) Photonic bandgap materials.

Hollow core photonic bandgap fibers (PBGFs), allowing light guidance in a low refractive index medium with tight mode confinement and ultra-low loss, have been paramount to the recent demonstration of high sensitivity gas spectroscopy cells [1], high energy soliton pulse delivery and compression [2] and efficient gas and liquid based nonlinear devices [3]. However, the transmission bandwidth of commercially available PBGFs, with a typical hole diameter to pitch ratio $(d / \Lambda)$ of $\sim 0.95-0.97$ and bandgap width to center transmission wavelength ratio $(W)$ of $\sim 10$ $15 \%$ or $100-200 \mathrm{~nm}$ at telecoms wavelengths, has limited their use to relatively narrow bandwidth applications. This has precluded further advances in areas such as, for example, fs pulse delivery, absorption and Raman spectroscopy or wide bandwidth gas/liquid-phase based nonlinear optics.

Significant theoretical and experimental efforts have been therefore devoted recently to identify the structural features responsible for bandgap formation and to maximize the bandgap width of fabricated PBGFs. It is now clear that out-of-plane photonic bandgaps (OOP-PBGs) originate from the interaction of coupled glass resonators (also known as rods or apexes), while the interconnecting glass struts necessary to support the structure have only the undesirable effect of narrowing down the overall PBG width [3]. For this reason, active pressurization techniques during the fiber draw have been introduced lately to increase the achievable air filling fraction in the cladding, thus reducing the strut thickness and ultimately increasing $W$. Using the conventional lattice generated by closely stacking circular capillaries, which will be referred to as the triangular lattice of holes (TLH), fibers with a $d / \Lambda$ close to 0.99 and $W \sim 33 \%$ have been now demonstrated [4]. At the same time, numerical studies have shown that a cladding formed by a square lattice (SL) of holes can generate normalized PBG widths $W$ more than $20 \%$ wider than the conventional TLH [5]. Fabrication of a SL PBGF with $d / \Lambda \sim 0.99$ and a record high $W \sim 44 \%$ has been recently reported [6].

In this Letter we demonstrate numerically that an improved cladding, based on a triangular lattice of rods (TLR) rather than holes, is able to generate significantly wider OOP 
bandgaps than any other known lattice. Based on this, we propose a realistically achievable lattice capable of generating an OOP bandgap spanning an octave $(W=66 \%)$, enabling the simultaneous transmission of a frequency and its second harmonic. Introducing a suitably constructed hollow core we finally design a realistic PBGF with sub- $\mathrm{dB} / \mathrm{m}$ confinement loss over a spectral range of $\sim 1000 \mathrm{~nm}$ in the near infrared.

This work stems from the observation that a possible explanation for the larger values of $W$ achievable in the SL as compared to the TLH lies in the different number of bandgap-opening resonating rods surrounding each hole: 6 in the TLH versus only 4 in the SL. As a consequence of this, for the same hole dimension in the SL the resonating rods are further apart. Their optical interaction is hence weaker and the observed bandgaps are wider, in analogy with the well known behavior of low index contrast, all-solid PBGFs [7]. If this physical intuition is correct, one would expect even wider bandgaps in a TLR, where each triangular-like hole is surrounded by only 3 resonators (see Fig.1). Although such a lattice has already been proved able to support air guidance [8], its performances have never been compared to those of other lattices.

Using a finite element method (FEM) with Bloch boundary conditions and a non-uniform mesh, the OOP-PBGs of all three lattices have been thoroughly compared. First the width along the air-line of the normalized OOP bandgaps ( $\left.W^{\prime}\right)$ generated by non interconnected circular silica rods in air, forming TLH, SL and TLR arrangements, were studied. Simulations showed that for the same hole diameter $(d)$ and hole-to-hole spacing $(\Lambda)$ the three lattices produced nearly identical values of $W^{\prime}$, suggesting that the differences exhibited by real lattices must be caused by the rod interconnections.

The performances of the three realistic lattices shown in Fig. 1 were then compared. The rods were joined by thin struts of thickness $T$ and the corners of the resulting holes were rounded with circular sections of radius $r_{c}$ to mimic the effect of surface tension. For each lattice type and a given value of $d / \Lambda=1-T / \Lambda$ a parametric scan in $r_{c}$ was conducted. Fig. 2(a) shows as an example the $d / \Lambda=0.98$ case. As can be seen, the PBG width is strongly dependent on the hole roundedness, and for each lattice the largest $W^{\prime}$ is obtained at an optimum value of $r_{c}$. In the structural parameterization adopted here, $r_{c}$ controls the size of the resonators relative to both hole size and strut thickness. If the resonators are too small (small $r_{c}$ ), their bandgaps are distorted and narrowed by the presence of the web of relatively thick interconnecting struts. Too large resonators (large $r_{c}$ ), on the other hand, interact too strongly amongst each other, again causing a reduction in the bandgap width. In between these two extremes lies the resonator size providing the maximum possible $W$ ' for a given lattice and $d / \Lambda$. It is worth pointing out that for all the $d / \Lambda$ studied, the lattices generating the widest bandgaps were found to be formed by rods of very similar size or cross-sectional area. Due to the different rod shape though, to achieve the same rod size, rather different values of $r_{c}$ are required in the different lattices (see Fig.1 and 2(a)).

Fig. 2(b) shows the largest possible value of $W$ ' for different values of $d / \Lambda$, ranging from the typical values of commercial fibers (0.95-0.98) to those of state-of-the-art fibers (0.99) and up to a not too unrealistic - but yet to be achieved - value of 0.995 . The results of these extensive simulations demonstrate that for moderate values of $d / \Lambda$, typical of current commercial fibers, the bandwidth generated by the TLR is almost twice that of the conventional TLH. For the more extreme $d / \Lambda=0.995$, not unforeseeable with further improvement in the fabrication process, the TLR with $r_{c}=0.114 \Lambda$ reaches the one octave limit $\left(W^{\prime}=66 \%\right)$, a $10 \%$ and $25 \%$ wider PBG than it is achievable with the best corresponding SL and TLH, respectively. 
This result can be explained by observing that if we fix $T$, the resonator size and the value of $T / \Lambda$, which is a structural constraint imposed by the fabrication process adopted, the distance between resonators $\left(\Lambda_{\mathrm{r}}\right)$ in the three lattices increases from $\Lambda / \sqrt{3}$ (TLH) to $\Lambda$ (SL) and $\sqrt{3} \Lambda$ (TLH) (see Fig.3). As already observed, the closer the optical resonators are and the stronger their interaction will be, resulting in a narrower spectral width of the antiresonance region, which in this case corresponds to the photonic bandgap [7].

It is also instructive to compare the performance of the 3 lattices having equal rod size and $d / \Lambda$, in the case where the rod separation $\left(\Lambda_{\mathrm{r}}\right)$, rather than the strut thickness $(T)$, is kept the same. Fixing the rod size produces the same long wavelength PBG edge, while if the same $\Lambda_{\mathrm{r}}$ and $d / \Lambda$ are imposed, the strut thickness progressively decreases going from TLH to SL and TLR. A thinner web of struts produces a less distorted and therefore wider bandgap, with the result that the short wavelength PBG edge of the TLR appears at shorter wavelengths than for the other lattices. Another consequence of keeping $\Lambda_{\mathrm{r}}$ constant is that the holes of the TLR become six times smaller than those of the TLH, therefore lowering considerably the effective index the air guided cladding modes [9]. As a result, the TLR bandgaps are not only wider, but also deeper, which suggests an increased resilience of these fibers to perturbation-induced losses such as micro and macro bend loss.

Next, using a full vector FEM modal solver with perfectly matched layers, the properties of a hollow core fiber based on the optimum TRL cladding with $\mathrm{d} / \Lambda=0.995$ and $r_{c}=0.114 \Lambda$ were investigated. A pitch $\Lambda_{\mathrm{r}}=4.3 \mu \mathrm{m}$ was chosen to center the bandgap around a wavelength of $1500 \mathrm{~nm}$; this structural condition fixes the required optimum rod diameter and strut thickness to $\sim 0.5 \mu \mathrm{m}$ and only $18 \mathrm{~nm}$, respectively. An air core formed by omitting one rod and its supporting struts was initially investigated, but was found to guide an air mode too close to the lower PBG edge, resulting in impractically high losses. A 7 missing rod core with a diameter of $\sim 17 \mu \mathrm{m}$ was therefore adopted. The simulated fiber structure and its calculated optical properties are shown in Fig. 4 and 5, respectively. With 6 and a half rings of rods, the fiber exhibits a confinement loss below $1 \mathrm{~dB} / \mathrm{m}$ in the spectral range $1035-2040 \mathrm{~nm}$, and below $0.1 \mathrm{~dB} / \mathrm{km}$ between 1050 and 1730 $\mathrm{nm}$, indicating a bandwidth of $1000 \mathrm{~nm}$ for device applications, and, potentially, of $680 \mathrm{~nm}$ for low loss telecoms data transmission. Additional rings of rods can further reduce the confinement loss across the full one octave bandwidth. Note that for this ideal, undistorted, geometry, the sudden termination of the periodic cladding around the core does not introduce any surface mode, in contrast to the case of conventional PBGFs where control of the core surround thickness is required [10]. The fiber dispersion is anomalous at wavelengths longer than 1120 $\mathrm{nm}$, and for central wavelengths sufficiently far from the bandgap edges, it presents a negligible third order contribution.

Similarly to the TLH case, a 7 missing element core results in a fiber which is moderately multimode in theory but effectively singlemode for long enough transmission lengths. For applications where strict single modality is mandatory however, a smaller core defect with only 3 missing rods may be the solution, provided one is prepared to accept the higher scattering losses associated with this choice [11].

In conclusion, we have studied a realistic lattice where resonating rods, rather than holes, are placed in a tightly packed triangular arrangement and are connected by ultra-thin struts. We have found that this alternative lattice, denominated TLR, consistently generates wider OOP bandgaps for a given $\mathrm{d} / \Lambda$ than both SL and TLH. With extreme but not unrealistic air filling fractions, it allows the simultaneous air-guidance of an octave of frequencies. Although more challenging to realize than the conventional TLH, the TLR is still amenable to fabrication by a 
modified stack and draw procedure, and its considerably wider bandgap can be worth the additional fabrication effort. The proposed ultrawide bandwidth fiber based on the TLR would be of interest to many applications in the areas of high energy ultrashort pulse generation and delivery, wide-bandwidth gas and liquid based nonlinear optical processes, gas spectroscopy and, potentially, telecoms transmission.

The author would like to thank P. Horak, M. N. Petrovich and D. J. Richardson for fruitful discussions and the Royal Society for support through a University Research Fellowship.

\section{References}

1. F. Benabid, F. Couny, J. C. Knight, T. A. Birks, and P. S. Russell, "Compact, stable and efficient all-fibre gas cells using hollow-core photonic crystal fibres," Nature 434, 488-491 (2005).

2. D. G. Ouzounov, F. R. Ahmad, D. Muller, N. Venkataraman, M. T. Gallagher, M. G. Thomas, J. Silcox, K. W. Koch, and A. L. Gaeta, "Generation of megawatt optical solitons in hollow-core photonic band-gap fibers," Science 301, 1702-1704 (2003).

3. F. Benabid, P. J. Roberts, F. Couny, and P. S. Light, "Light and gas confinement in hollowcore photonic crystal fibre based photonic microcells," Journal of the European Optical Society 4, (2009).

4. P. S. Light, F. Couny, Y. Y. Wang, N. V. Wheeler, P. J. Roberts, and F. Benabid, "Double photonic bandgap hollow-core photonic crystal fiber," Optics Express 17, 16238-16243 (2009).

5. F. Poletti and D. J. Richardson, "Hollow-core photonic bandgap fibers based on a square lattice cladding," Optics Letters 32, 2282-2284 (2007).

6. B. K. Thomas, S. Suzuki, L. Fu, and L. Dong, "First demonstration of photonic bandgap guidance in hollow core photonic bandgap fibers with square lattice cladding," in ECOC 2009 (postdeadline).

7. T. A. Birks, G. J. Pearce, and D. M. Bird, "Approximate band structure calculation for photonic bandgap fibres," Optics Express 14, 9483-9490 (2006).

8. M. Yan, P. Shum, and J. Hu, "Design of air-guiding honeycomb photonic bandgap fiber," Optics Letters 30, 465-467 (2005).

9. E. A. J. Marcatili and R. A. Schmeltzer, Bell Syst. Tech. J. 43, 1783 (1964).

10. R. Amezcua-Correa, N. G. R. Broderick, M. N. Petrovich, F. Poletti, and D. J. Richardson, "Optimizing the usable bandwidth and loss through core design in realistic hollow-core photonic bandgap fibers," Optics Express 14, 7974-7985 (2006).

11. M. N. Petrovich, F. Poletti, A. van Brakel, and D. J. Richardson, "Robustly single mode hollow core photonic bandgap fiber," Optics Express 16, 4337-4346 (2008). 

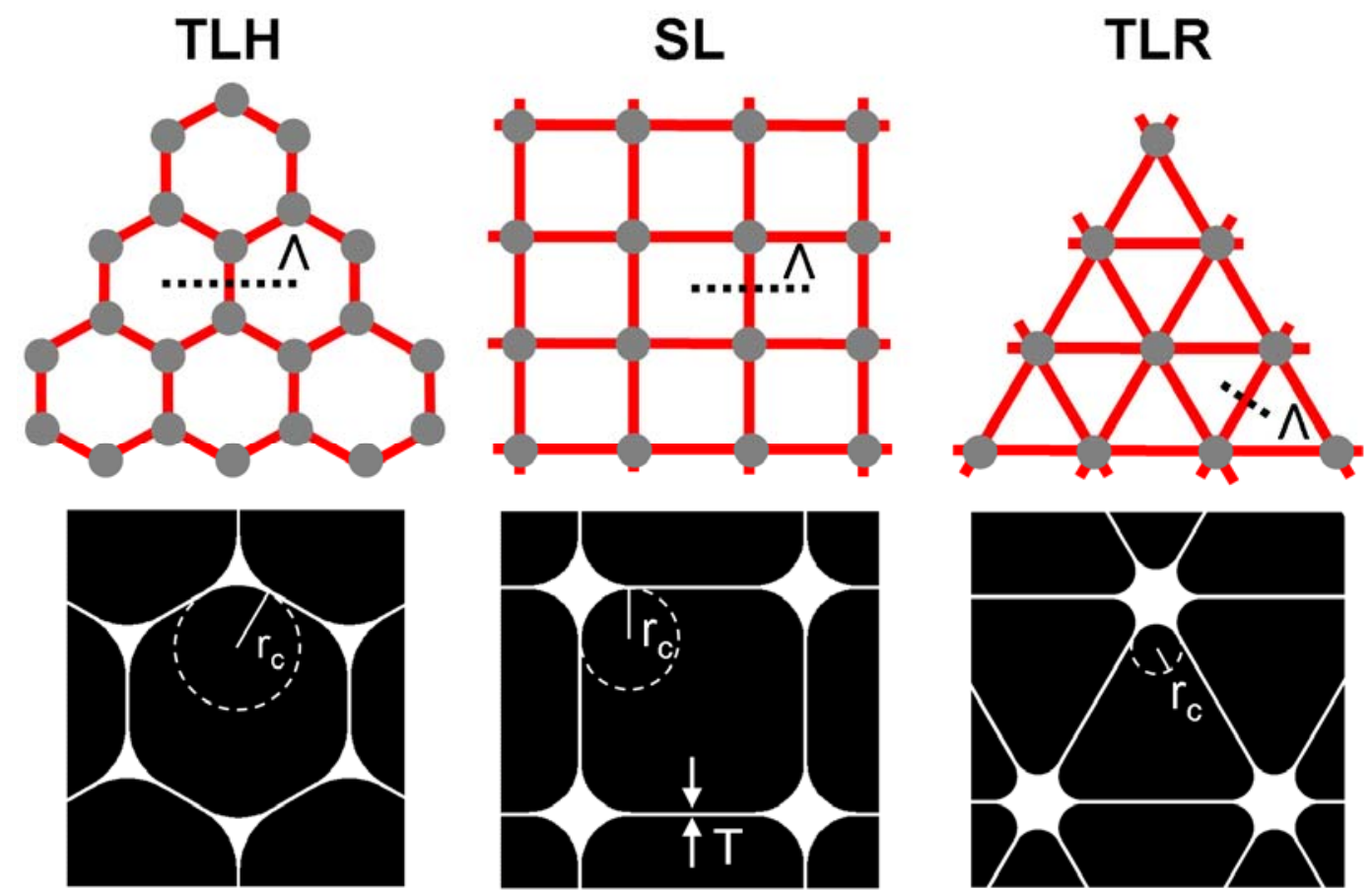

Fig. 1. (Color online) (Top) interconnected two dimensional lattices studied in this work: triangular lattice of holes (TLH), square lattice (SL) and triangular lattice of rods (TLR). Gray circles represent the resonating rods, connected by the red struts; (Bottom) hole shape of realistic claddings, with rounded holes where black and white areas represent air and silica, respectively.

(a)

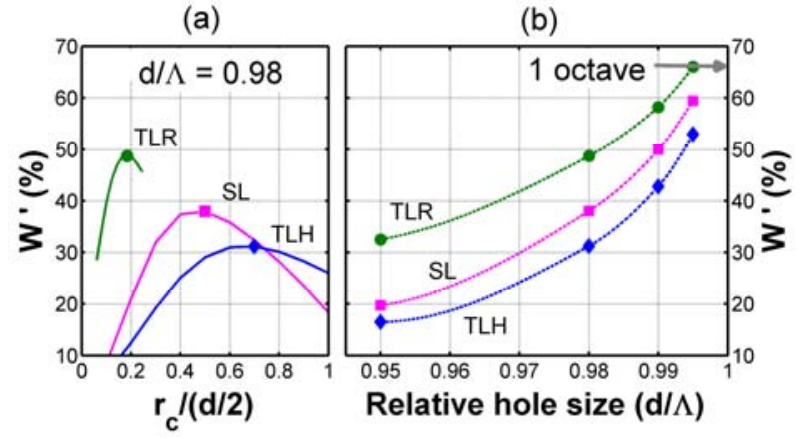

Fig. 2. (Color online) Width along the air line of the OOP fundamental PBG ( $\left.W^{\prime}\right)$ for the three lattices shown in Fig.1. (a) Dependence of $W^{\prime}$ on $r_{c}$, which controls hole roundedness and rod size, for $d / \Lambda=0.98$; (b) dependence of $W^{\prime}$ on $d / \Lambda$. Each point in this graph represent the maximum $W^{\prime}$ that can be achieved by optimizing $r_{c}$. 


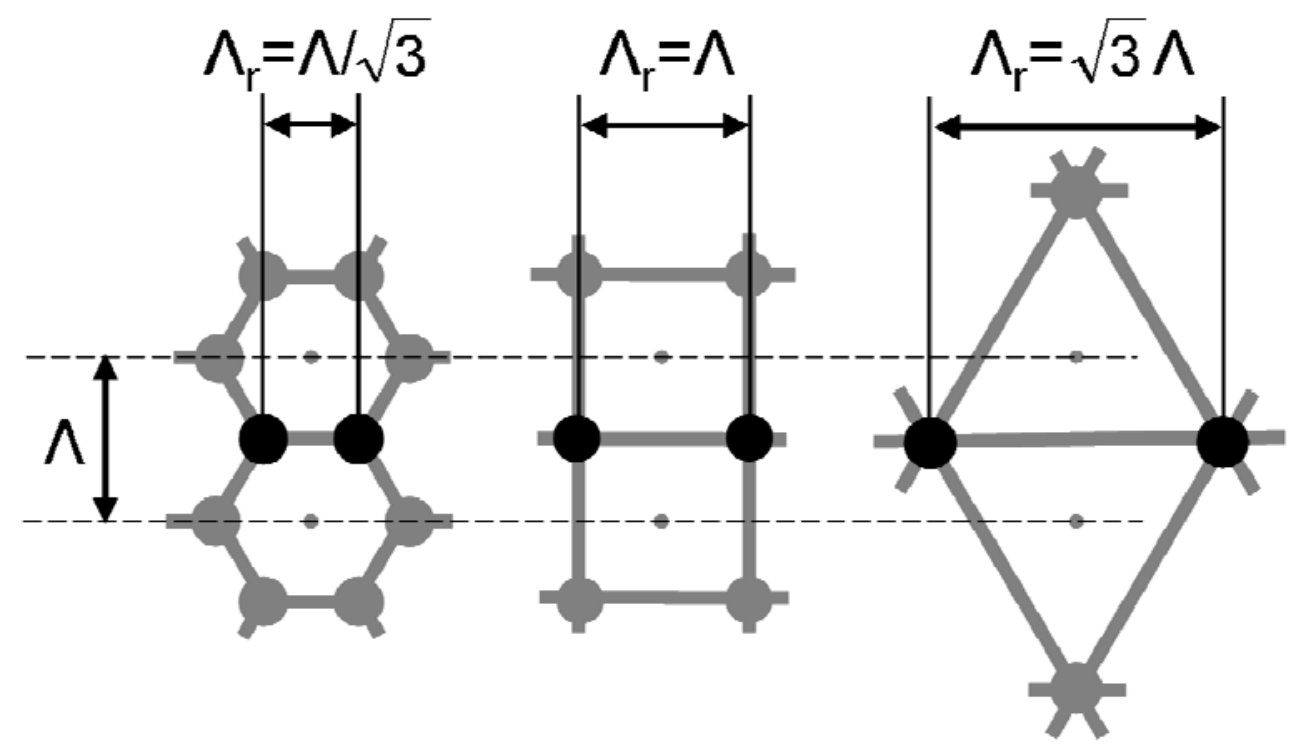

Fig. 3. Minimum rod spacing $\left(\Lambda_{\mathrm{r}}\right)$ versus hole-to-hole spacing $(\Lambda)$ for the three lattices of this study, from left to right: TLH, SL and TLR.

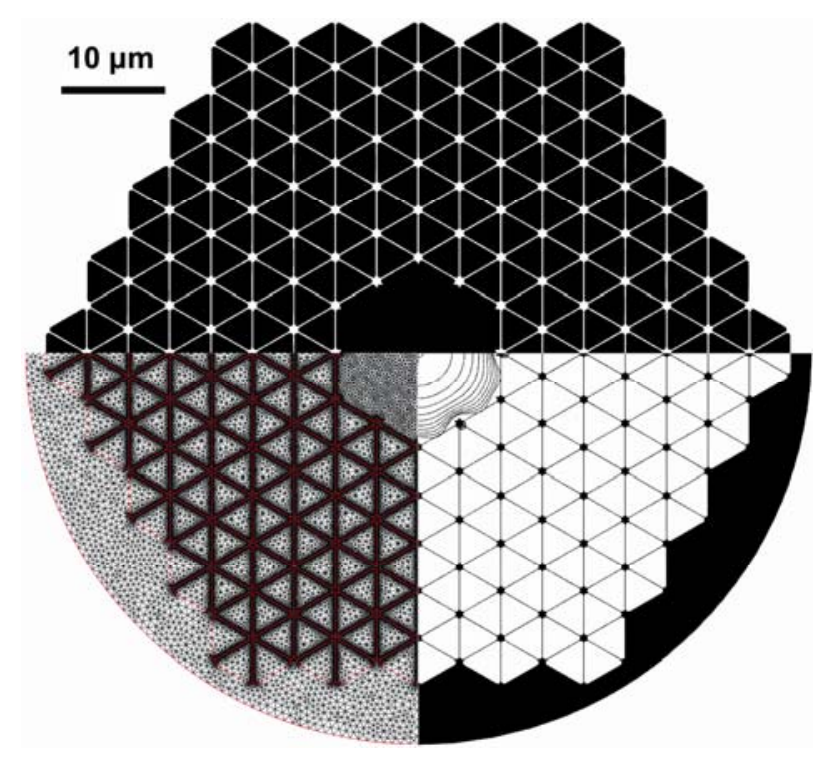

Fig. 4. Ideal structure of the octave spanning PBGF based on the TLR lattice. $d / \Lambda=0.995, \Lambda_{\mathrm{r}}=$ $4.3 \mu \mathrm{m}, r_{c}=0.114 \Lambda$. The fiber has a 7 missing rod central core and 6 and a half complete rings of rods. The mesh used in the FEM simulations (750.000 degrees of freedom) and the fundamental optical mode ( $2 \mathrm{~dB}$ contour lines) are also shown. 

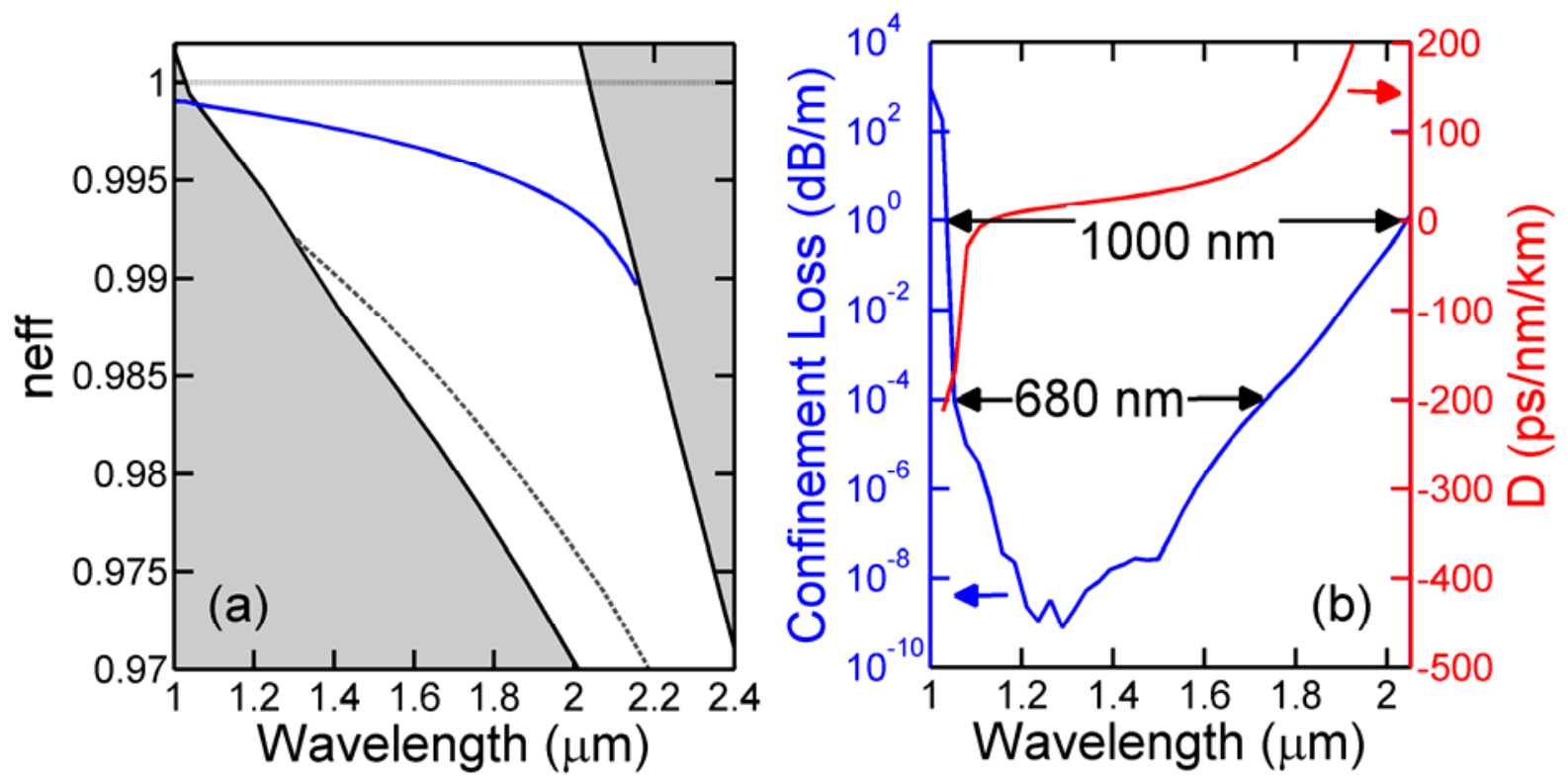

Fig. 5. (Color online) Simulated optical properties of the fiber in Fig. 3. (a) PBG of the optimum TLR cladding (white area) and effective index (neff) of the fundamental mode (solid line) and high order mode (dashed line); (b) Confinement loss and group velocity dispersion of the fundamental mode. 